7. Францевич И.Н. и др. Упругие постоянные и модули упругости металлов и неметаллов. Справочник. - Киев: Наукова Думка, 1982. - 286 с.

8. Хантингтон Г. Упругие постоянные кристаллов // УФН. - 1961. - T. LXXIV, вып. 2. - C. $303-352$.

9. Хачин В.Н., Муслов С.А., Пушин В.Г., Кондратьев В.В. Особые упругие свойства В2-соединений титана с нестабильной решеткой // Металлофизика и новейшие технологии. - 1988. - Т. 10. - № 1. C. 102-104.

10. R.N. Thurston and K. Brugger. Third-Order Elastic Constants and the Velocity of Small Amplitude Elastic Waves in Homogeneously Stressed Media. Phys. Rev., 133, A1604 (1964).

11. Zung-Ping Chang. Third-Order Elastic Constants of $\mathrm{NaCl}$ and $\mathrm{KCl}$ Single Crystals. Physical Review, 1965 vol. 140, Issue 5A, pp. 1788-1799.

\title{
Степанец B.A.
}

\section{К вопросу о природе электрического поля}

АО «Научно-производственный испьтательный иентр «Арминт» (Россия, Москва)

doi:10.18411/spc-16-01-2018-03

idsp: 000001:spc-16-01-2018-03

«Нет ничего практичнее хорочей теории» Д.И. Менделеев

\section{1.Введение}

Расчеты футурологов показывают, что без нового мощного источника «чистой» энергии нельзя выйти из надвигающегося энергетического кризиса и разрешить многочисленные нарастающие проблемы экологии и глобального изменения климата. И потребность в нем только растет.

Известные виды относительно «чистой» энергии: гидравлической, ветровой, геотермальной, солнечной, ядерной ресурсно ограничены. Промышленное использование термоядерной энергии пока видится в отдаленной перспективе. В то же время мы «купаемся» в море окружающей и атакующей Землю энергии космических лучей, нейтринного излучения, «темной» энергии, гравитационных, электрических и других полей...

Хотелось бы научиться утилизировать все эти энергии. Начать представляется целесообразным с наиболее исследованной из них - энергии электрического поля, с которым человечество знакомо порядка трех тысяч лет. Причем непонимание сути электричества не помешало его широкому практическому применению. Вместе с тем, не вызывает сомнений, что использование электрической энергии на основе полного знания физики электрических процессов было бы гораздо эффективнее.

\section{2.История познания электричества}

Анри Ампер указывал, что все электрические явления производит взаимодействие (притяжение и отталкивание) молекул электрических жидкостей. Эфир представляет собой соединение двух электрических жидкостей, разложение и соединение которых образуют электрический ток, являются единственной причиной образования тепла и света, т.е. колебаний жидкости, заполняющей все пространство [1]. Майкл Фарадей оставлял возможность многовариантности природы передачи электрических сил (электростатической индукции), ставя своей задачей дать описание результатов их действия, основываясь исключительно на опытных данных. Разрабатывая теорию силовых линий, он, в частности, допускал передачу этих сил, как через смежные частицы вещества, так и через вакуум [2].

Альберт Эйнштейн определял электрическое поле как нечто, обладающее энергией, помещающееся между заряженными телами, чтобы описать действующую между ними силу [3]. В теории электричества у Николы Теслы основополагающим было понятие эфира - некой невидимой субстанции, заполняющей весь мир и 
передающей колебания со скоростью, во много раз превосходящей скорость света. Каждый миллиметр пространства, полагал Тесла, насыщен безграничной, бесконечной энергией, которую нужно лишь суметь извлечь [4].

«Баллистическая теория» Вальтера Ритца [5] привлекательна предельной простотой, отказом за ненадобностью и бесполезностью от понятия магнитного поля и постулатов Эйнштейна. Отталкиваясь от фотонной (корпускулярной) теории истечения света Ньютона, основываясь на конечности скорости распространения любых материальных объектов, Ритц свел все известные электромагнитные явления к механическим взаимодействиям зарядов посредством излучаемых ими «фиктивных» (виртуальных) частиц.

В классической электромагнитной теории устоявшимся является следующее определение электрического поля [6]: «особый вид материи, создаваемый в окружающем пространстве заряженными частицами или телами, посредством которого осуществляется взаимодействие между этими заряженными частицами или телами». Процесс возникновения и исчезновения поля и его природа при этом остаются за рамками теории. Силовой характеристикой электрического поля служит вектор его напряженности E: E = F/q, где F - сила, действующая на заряд q.

Согласно современной квантовой теории поля, взаимодействие поля и заряда, а также свободных зарядов между собой, происходит в форме испускания и поглощения зарядами квантов поля - фотонов. Например, между двумя электронами идет постоянный обмен фотонами: каждый из электронов испускает фотоны (кванты) электромагнитного поля, которые затем поглощаются другим электроном. Классическое же взаимодействие в виде сил, действующих между заряженными частицами, оказывается вторичным эффектом, возникающим в результате обмена виртуальными квантами поля, переносящими взаимодействие [7]. Заметим, что утверждение о возникновении электрических сил в результате обмена виртуальными низкочастотными квантами абстрактно и недостаточно для понимания физики превращения элементарных обменных актов в силовое воздействие.

В порядке компромисса ныне принята дуальная модель электромагнитного поля - классическая волновая в макроявлениях и корпускулярная (квантовая) в микромире.

\section{3.Необходимость в новых теориях электрического взаимодействия}

На настоящее время накоплены десятки экспериментов, свидетельствующих о некорректности классической электромагнитной теории и ее несоответствии картине реальных физических явлений: опытным данным, принципу причинности и естественности в смысле соответствия общечеловеческой практике (см., например, [8]). В качестве примера, а также в целях выявления возможного перспективного направления исследований, рассмотрим один из конфликтов теории электромагнитного поля и классической ньютоновской механики.

Как известно, взаимодействие движущихся зарядов проходит с нарушением принципа центральности сил, третьего закона Ньютона и, соответственно, закона сохранения механического импульса (например, см. статью «Электродинамика» [9] Физической энциклопедии, работы Р. Фейнмана [10], И. Савельева [11]). В современной электродинамике, для формального выполнения закона сохранения импульса, образовавшуюся разницу в расчете механического импульса относят на долю импульса электромагнитного поля (заметим, что в «баллистической теории» Ритца такого несоответствия нет). Ниже будет показано, что и в электростатике постулируемые принципы центральности и суперпозиции сил и полей также не выполняются.

Но если сумма внутренних сил механической системы (независимо от их природы), приложенных к элементам этой системы, не равна нулю, то из этого факта автоматически следует образование равнодействующей силы и принципиальная возможность организации так называемого безопорного движения! 
Таким образом, возникает необходимость в адекватном непротиворечивом теоретическом описании известных электрических явлений. Руководствуясь рекомендацией Д.И. Менделеева: «Из всех гипотез выбирайте ту, которая не пресекает дальнейшего мышления об исследуемых вещах», предпочтение следует отдавать теоретическим положениям, непосредственно основанным на опытных данных. С позиции максимы Уильяма Оккама: «Не следует привлекать новые сущности без крайней на то необходимости», следует признать преимущества «баллистической теории» Ритца, основанной на простых, понятных и естественных механизмах. В то же время не вызывает сомнений ряд подтвержденных экспериментально аналитических соотношений электростатики и квантовой теории поля.

Из изложенного вытекает задача поиска нового, не противоречащего известным знаниям об электричестве пути дальнейшего продвижения вперед. Попытаемся, без потери физического смысла и содержания теоретических предположений сформулировать несколько возможных подходов для ее решения.

\section{4.Физическая сущность электрического поля}

Гипотеза существования потока электрического поля. Допустим, имеется еще некоторое (дополнительно к трем, эмпирически известным человечеству) количество измерений материального мира. Пусть одно из них сопрягается с нашей (ощущаемой людьми) Вселенной через точки (проколы, порталы), являющиеся положительными и отрицательными зарядами. И пусть это измерение проявляется в существовании у положительных и отрицательных зарядов потока материальной энергии, пропорционального значениям этих зарядов. Назовем его потоком электрического поля или электрополевым потоком.

Попробуем, основываясь на опытных данных и известных физических эффектах, определить характеристики и свойства этого потока.

1). Формируемый (вероятно в виде вихря) между разноименными зарядами вещества электрополевой поток действует постоянно, обеспечивая удержание электронов в составе атомов. Он же существует и в макромире, взаимодействуя со свободными зарядами в процессе испускания ими или поглощения субстанции (материальных частиц) потока.

2). Представляется принципиально возможным, что элементарные частицы электрон и позитрон являются как излучающе-поглощающими, так и чисто поглощающими субъектами электрополевого потока, а предельно сблизившиеся пары электрон-позитрон образуют нейтрино. При этом поток может испускаться и поглощаться не только непрерывно, но и квантами (фотонами) при совершении электронами межорбитальных переходов.

3). Электрополевой поток обладает плотностью, а его частицы, которые также можно назвать элементами наполняющего пространство «эфира», массой. Частицы потока, подобно молекулам стандартного газа, отталкиваются друг от друга, стремясь к максимальному рассеиванию. Это следует из опытов электростатики [12], в которых напряженность (интенсивность) поля заряженного тела произвольной формы или нескольких тел, по мере удаления от них, постепенно усредняется и, начиная с некоторого расстояния, становится равномерно сферической (см. Рис. 1, 2). Это очевидно и из опытов Фарадея [13], выявившего явление обтекания полем препятствий, а также, измерений давления света, отклонения лучей света под гравитационным воздействием и др.

4). Измерения силы, действующей на пробный заряд в однородном поле, показывают, что она не зависит от расстояния до источника или стока поля, а определяется только интенсивностью (плотностью) электрополевого потока этого поля и величиной пробного заряда. Таким образом, закон Кулона определяет не столько силу, сколько плотность электрополевого потока в контрольной точке или, в терминах классической электростатики, степень рассеяния поля заряда. 


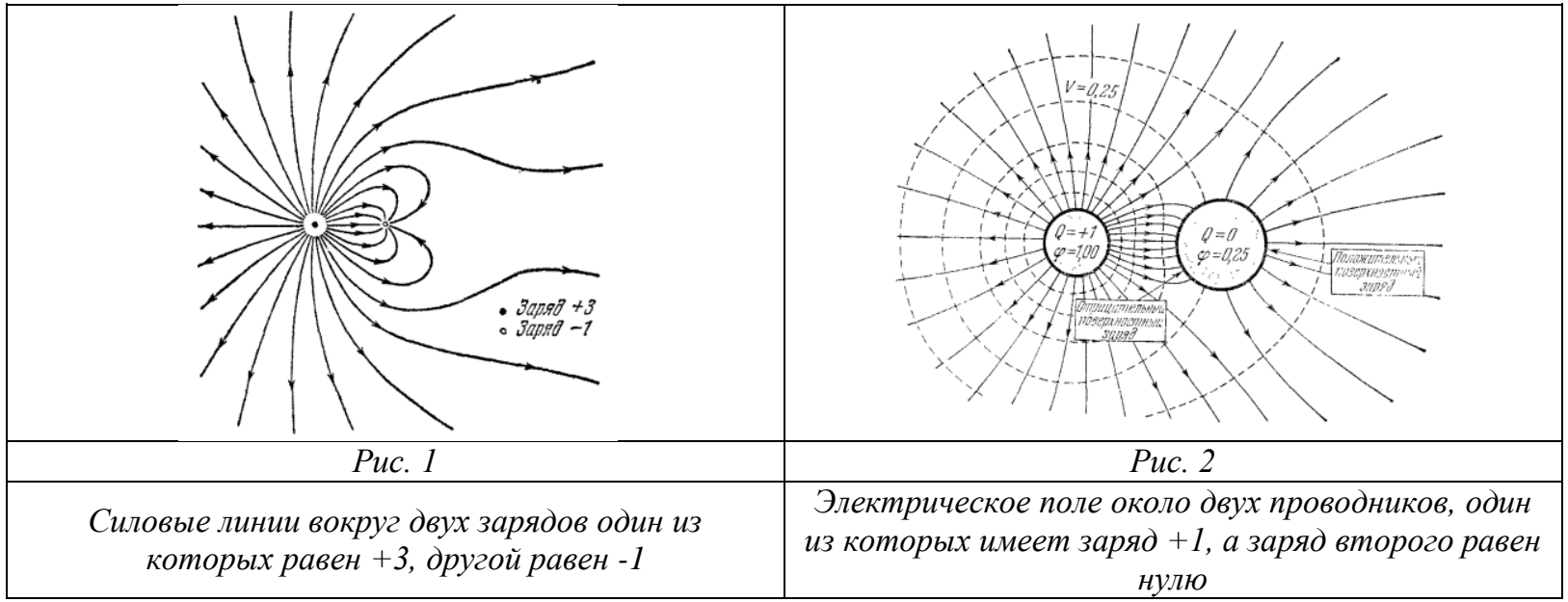

5). Из характера действующих на поверхности заряженных уединенных проводников электростатических сил [14], которые всегда направлены наружу, нормально к поверхности проводника, следует, что поглощение или испускание субстанции потока поля происходит с усилием, приложенным к элементарным зарядам и суммарно к заряженному телу в целом. Результирующая сила этого распределенного усилия направлена по градиенту возрастания интенсивности электрополевого потока (напряженности поля) вокруг этого заряда или тела. Определить значение результирующей силы можно просуммировав (проинтегрировав) векторы силы линий поля на поверхности заряда или заряженного тела (см. Рис. 3).

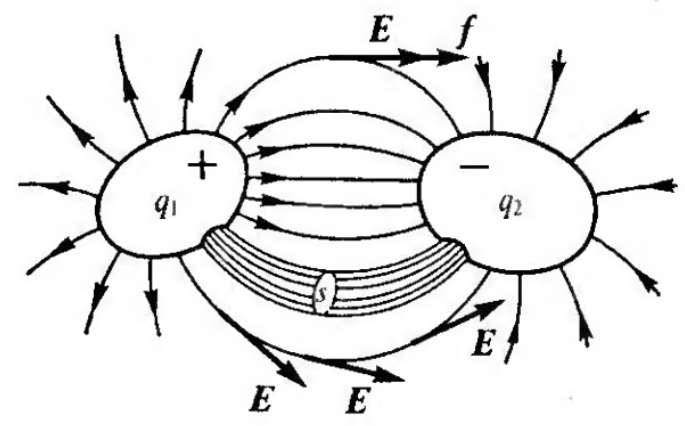

Puc. 3

Карта силовых линий поля двух разноименно заряженных проводников

6). Скорость распространения фотонного излучения потока электрического поля в вакууме равна скорости света, что, видимо, связано с особенностями механизма их испускания, и аддитивно складывается со скоростью источника излучения согласно законам классической механики, что доказывается эффектом «красного» и «синего» смещения спектра излучения движущегося источника. Характер непрерывного течения частиц потока, как показывают электрические карты сложных полей (см. Рис. 1-3), определяется кинематическими параметрами действующих потоков и может происходить с любой скоростью, от световой до нулевой, а остановившиеся частицы поля, «стянутые» гравитацией галактик, наряду со слабовзаимодействующими частицами типа нейтрино, возможно, составляют «темную» материю. Также логично предположить, что циркулирующие во Вселенной фотонные электрополевые потоки, испускаемые в виде света и других видов излучения, например, объектов типа «белых дыр», оказывая давление на материю, как раз и ответственны за «разбегание» галактик.

\section{5.Электрополевая энергетика}

Что дает существование потока электрического поля практически? В теоретическом плане, для адекватного описания взаимодействия зарядов становится возможным использовать математический аппарат гидроаэромеханики и молекулярной 
физики. Но самое главное, понимание существования в нашем материальном мире этих электрополевых потоков обеспечивает принципиальную возможность использования их кинетической энергии как неисчерпаемого внешнего источника энергии.

Одним из направлений практического применения выдвинутой гипотезы является построение считающихся ныне невозможными безопорных движителей. По физике функционирования, такие движители являются электрическими машинами, преобразующими энергию электрического поля (электрополевого потока), действующего между механически связанными между собой разноименно заряженными электродами устройства, в механическую энергию их взаимного вращательного или поступательного движения. Рассмотрим возможный вариант такого конструирования на примере устройства, заявленного в качестве изобретения: «Способ электромеханического преобразования энергии и электрополевой движитель на его основе».

Будем отталкиваться от известного. В электростатике хорошо изучены характеристики поля плоского электрического конденсатора [15]. Действующие между его заряженными обкладками силы наглядно описываются совокупностью силовых линий. В однородной части поля конденсатора они равны, встречно направлены, центральны и в целом взаимно компенсируют друг друга (см. Рис. 5, 6).

\begin{tabular}{|c|c|}
\hline 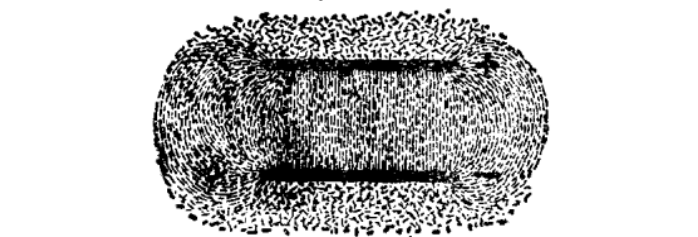 & + \\
\hline Puc. 4 & Puc. 5 \\
\hline $\begin{array}{c}\text { Расположение крупинок между разноименно } \\
\text { заряженными пластинами }\end{array}$ & $\begin{array}{c}\text { Линии поля между разноименно заряженными } \\
\text { пластинами }\end{array}$ \\
\hline
\end{tabular}

Расположение крупинок между Линии поля между разноименно разноименно заряженными пластинами заряженными пластинами

Посмотрим, что получится, если развести обкладки конденсатора на некоторый угол.

В теории известно описание электростатического поля для двух проводящих заряженных плоскостей, сходящихся под углом [16] (см. Рис. 6).

\begin{tabular}{|l|}
\hline Рис. 6 \\
\hline Поле плоских разноименно заряженных \\
проводников, развернутьх под углом
\end{tabular}

Линии равной напряженности (силовые линии) этого поля представляют собой окружности, проведенные из вершины двугранного угла, образованного плоскостями, на которых располагаются заряженные проводящие плоскости. А величина напряженности Е является функцией разности потенциалов $\mathrm{U}=\mathrm{U} 2$ - U1 и длины силовой линии между электродами: 


$$
\mathrm{E}=\mathrm{U} /(\alpha \cdot \mathrm{r}),
$$

где, $\alpha$ - угол между плоскостями [рад];

$\mathrm{r}$ - радиус силовой линии.

Расчеты показывают, что в отличие от плоского конденсатора, суммарные силы между двумя заряженными плоскими проводящими плоскостями 1,2 , зафиксированными диэлектрическими опорами 3 под углом, не компенсируются (см. Рис. 7).

Назовем эту систему плоских проводников, характерную переменным (расходящимся) расстоянием между пластинами, «конденсаторным диполем» или «раскрывающимся конденсатором». Поскольку общий вектор приложенной к каждой пластине силы формируется силовыми линиями на поверхности пластин, то, таким образом, в системе заряженных обкладок (пластин) конденсатора имеем некоторую результирующую силу, направленную по биссектрисе угла между пластинами конденсатора, которая формируется следующим образом.

Будучи заряженными, элементарные участки пластин конденсаторного диполя притягиваются друг к другу с силой $f 1,2$, результирующая векторной суммы которых в проекции на срединную линию устройства дает линейную составляющую fл:

$$
\mathrm{f}_{\text {л }}=\mathrm{f} \cdot \sin (0,5 \alpha)
$$

где $\mathrm{f}=|\mathrm{f} 1|+|\mathrm{f} 2|$ - алгебраическая сумма модулей сил $\mathrm{f} 1$ и f2.

Проинтегрировав указанные электростатические силы по площади пластин, получаем приложенную к конденсаторному диполю результирующую силу Fp, которая может быть использована для выполнения полезной работы.

С учетом формул связи напряженности Е и плотности $\sigma$ заряда, а также действующей на поверхности пластины шириной $\mathrm{h}$ и длиной (rк - ro) силы $\mathrm{F}$, в условиях вакуума [17], равнодействующая результирующая сила Fр составит:

$$
\mathrm{F}_{\mathrm{p}}=2 \mathrm{~F} \cdot \sin (0,5 \cdot \alpha)=\varepsilon_{\mathrm{o}} \cdot \mathrm{U}^{2} \cdot \mathrm{h} \cdot \sin (0,5 \cdot \alpha) \cdot\left(1 / \mathrm{r}_{\mathrm{o}}-1 / \mathrm{r}_{\mathrm{K}}\right) / \alpha^{2}
$$

Расчеты по приведенным выше соотношениям показывают, что для тонких пластин, развернутых на острый угол, действующими между обратными сторонами пластин, а также между краями устройства силами можно пренебречь.

Таким образом, мы показали, что не только в динамике, но и в статике принципы суперпозиции и центральности электрических сил и полей в общем случае не соблюдается.

Для выполнения функции движителя указанные обкладки располагают в диэлектрической среде с малой диэлектрической проницаемостью и разворачивают друг относительно друга таким образом, чтобы образующаяся при этом между обкладками результирующая сила электростатического взаимодействия была ориентирована в направлении требуемого их перемещения. Т.е. получаем универсальный безопорный (в обычном понимании этого слова) электрополевой движитель, преобразующий энергию электрического поля в энергию механического движения.

Закрепив указанные пластины или комбинацию их пар на рабочем объекте (теле), которое необходимо вращать или перемещать в пространстве, совершаем требуемую полезную работу. Необходимая сила взаимодействия заряженных обкладок регулируется изменением величины их заряда любым известным методом, например, от источника регулируемого напряжения.

Работоспособность заявленного способа подтверждена экспериментально, его новизна и изобретательский уровень закреплены патентом РФ за № 2629846,. На этих же принципах построены электрополевые двигатели по патентам РФ № 2458451 и № 2471283 и генераторы электроэнергии по патентам РФ № 2471284 и № 2454783. 


\section{6. Заключение}

В заключение немного истории. Бурное развитие электрополевой энергетики в девятнадцатом веке привело к созданию многочисленных образцов электрополевых машин - электростатических генераторов и двигателей. К сожалению, в дальнейшем они не выдержали конкуренции с машинами, основанными на эффекте электромагнитной индукции, и забылись.

Настало время возвращения к истокам электростатического машиностроения, но уже на новом уровне идей электрополевой энергетики. Кроме того, заметим, что возможность получения энергии из физического вакуума, как сложной энергетической системы со свернутыми в подпространствах вакуума кольцевыми электрическими структурами вещества-антивещества уже рассматривалась теоретически, например, советским физиком и математиком Герловиным И.Л. в разработанной им теории фундаментального поля [18]. Заявления астрофизиков о существовании «темной» материи и «темной» энергии также говорят в пользу выдвинутой гипотезы существования потока электрического поля.

Актуальность и возможные прикладные аспекты электрополевой энергетики в решении ряда практических задач рассмотрены в статье «Электрополевая энергетика: постановка задачи» [19].

Автор выражает глубокую благодарность кандидату физико-математических наук Гаврилову С.Д. за участие в обсуждении и подготовке материалов статьи к публикации.

$$
* * *
$$

1. Ампер А М Электродинамика (М.: Изд. АН СССР, 1954) с.194, 288-289.

2. Фарадей М Избранные работы по электричеству (М.-Л.: ГОНТИ, 1939) с.193-196.

3. Эйнштейн А Эволюция физики (М.: ООО «Тайдекс Ко», 2005) с. 134-139, 144-146, $209-212$.

4. Тесла Н Лекции и статьи (М.: «Tesla Print», 2003) c.23-25.

5. Семиков С А Баллистическая теория Ритца и картина мироздания (Н. Новгород: ООО «СтимулCT», 2010).

6. Яворский Б М, Детлаф А А Справочник по физике (М.: «Наука», 1974) с.343.

7. Савельев И В Курс общей физики (СПб.: «Лань», 2008) Т.3, с.239-244.

8. Николаев Г В Современная электродинамика и причины ее пародоксальности. Перспективы построения непротиворечивой электродинамики (Томск: Изд-во «Твердыня», 2003).

9. Кочаровский В В, Кочаровский Вл В в сб. Физическая энциклопедия (Гл. ред. А М Прохоров) (М.: Советская энциклопедия, 1988) Т.5, с.519-531.

10. Фейнман Р Ф и др. Фейнмановские лекции по физике (М.: Издательство ЛКИ, 2010) Вып.6, с.270, 303.

11. Савельев И В Курс общей физики (СПб.: «Лань», 2008) Т.1, с.60-62.

12. Парселл Э М Курс общей физики (М.: Наука, 1971) Т.2, с. 36, 103.

13. Фарадей М Экспериментальные исследования по электричеству (М.: Изд. АН СССР, 1947) Т.1, с. 503-512.

14. Тамм И Е Основы теории электричества (М.: Наука, 1989) с. 68-69.

15. Ландсберг Г С и др. Элементарный учебник физики (М.: Физматлит, 2008) Т.2, с.24-27, 71-74.

16. Демирчян К С и др. Теоретические основы электротехники (СПб.: Питер, 2004) Т.3, с.51-52.

17. Яворский Б М, Детлаф А А Справочник по физике (М.: Наука, 1974) с.357, 358.

18. Герловин И Л Основы единой теории всех взаимодействий в веществе (Л.: Энергоатомиздат, 1990) c. 12-15.

19. 19. Гаврилов С Д, Степанец В А Проблемы безопасности и чрезвычайных ситуаций $\quad$ (№ 2, 2014) c.96-105. 